BECKER, L.; FURTINI NETO, A.E.; PINTO, J.E.B.P.; CARDOSO, M. G.; SANTOS, C.D.; BARBOSA, J.M.; LAMEIRA, O A.; SANTIAGO, E.J.A. Crescimento e produção de alcalóides totais de quebra-pedra em função da calagem e da adubação nitrogenada. Horticultura Brasileira, Brasília, v. 18, n. 2, p.100 - 104, julho 2000

\title{
Crescimento e produção de alcalóides totais de quebra-pedra em função da calagem e da adubação nitrogenada.
}

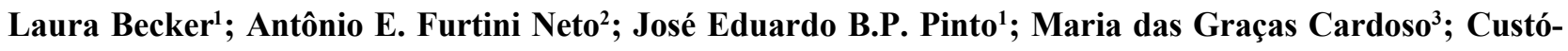 \\ dio D. Santos ${ }^{3}$; Janaynna M. Barbosa ${ }^{1}$; Osmar A. Lameira ${ }^{4}$; Edson J. A. de Santiago ${ }^{4}$ \\ ${ }^{1}$ UFLA - Dept $^{\mathrm{o}}$ Agricultura, ${ }^{2}$ UFLA - Dept ${ }^{\underline{0}}$ Ciências dos Solos, ${ }^{3}$ UFLA - Dept ${ }^{\circ}$ Química, C. Postal 37, 37.200-000 Lavras - MG; \\ ${ }^{4}$ Embrapa Amazônia Oriental, Belém - PA.
}

\section{RESUMO}

Foram cultivadas, em casa-de-vegetação, cinco plantas de Phyllanthus niruri $\mathrm{L}$ em vasos com volume de $3,4 \mathrm{dm}^{3}$, tendo como substrato um Cambissolo Álico, com textura média, coletado no município de Nazareno - MG. As sementes de quebra-pedra foram oriundas de Belém - PA. Os tratamentos consistiram de cinco doses de $\mathrm{N}\left(0,30,60,100\right.$ e $\left.150 \mathrm{mg} / \mathrm{dm}^{3}\right)$ e o uso ou não de calcário, sendo que nos tratamentos com calcário visava-se a elevação do $\mathrm{pH}$ para 6,0. O delineamento experimental foi inteiramente casualisado com seis repetições. As plantas foram colhidas aos oitenta dias de ciclo, quando foi avaliado o peso da matéria seca da parte aérea e da raiz e efetuadas as análises químicas e fitoquímicas da parte aérea das plantas. O quebra-pedra é uma espécie responsiva à calagem e à adubação nitrogenada, apresentando na dose de nitrogênio de $77,81 \mathrm{mg} /$ $\mathrm{dm}^{3}$ de solo o equivalente a $90 \%$ da produção máxima média $(12,25$ $\mathrm{g} / \mathrm{vaso}$ ) de matéria seca total, quando na presença de calagem. $\mathrm{Na}$ ausência de calagem a produção de matéria seca total decresce linearmente (de 3,9 g/vaso a 2,0 g/vaso) com o aumento das doses de N. O teor de $\mathrm{P}, \mathrm{S}$, Ca e Mg nas plantas foi maior nos tratamentos com calagem, independente das doses de N. Na ausência de calagem, o conteúdo dos macronutrientes na matéria seca total foi menor em conseqüência da menor produção de matéria seca total. na presença de calagem. Na presença de calagem, observou-se aumento na matéria seca da parte aérea e na concentração de alcalóides à medida que se aumentaram as doses de nitrogênio aplicadas. A máxima produção de alcalóides totais foi de $0,676 \mathrm{mg} / \mathrm{g}$ de matéria seca total da parte aérea, que corresponde à dose de $\mathrm{N}$ equivalente a $90 \%$ para máxima produção de matéria seca da parte aérea.

Palavras-chave: Phyllanthus niruri L, adubação, nutrição mineral.

\begin{abstract}
Growth and production of total alkaloids of 'quebra-pedra' as a result of the use of lime and nitrogen fertilization.

Five plants of Phyllanthus niruri L. were cultivated in a greenhouse in $3.4 \mathrm{dm}^{3}$ pots with Alic Cambisol (medium texture) as substrate. Soil samples were collected in Nazareno - MG and seeds from 'quebra-pedra' were originated from Belém, Brazil. The treatments consisted of five concentrations of $\mathrm{N}\left(0,30,60,100\right.$ and $150 \mathrm{mg} / \mathrm{dm}^{3}$ of $\mathrm{N}$ ), two levels of liming (with and without limestone, aiming at raising the $\mathrm{pH}$ to 6.0 ). The experimental design was completely randomized with 6 replications. Plants were harvested eighty days after transplanting. Root, stem and leaves dry matter production were evaluated and chemical and phytochemical analysis of stems and leaves were performed. 'Quebra-pedra' is a plant species responsive to liming and nitrogen fertilization showing, in the presence of liming, the equivalent to $90 \%$ of the maximum total dry matter yield $(12.25 \mathrm{~g} /$ pot) for the nitrogen level of $77.81 \mathrm{mg} / \mathrm{dm}^{3}$ of soil. In the absence of liming the total dry matter decreased linearly (from $2.0 \mathrm{~g} / \mathrm{pot}$ to $3.9 \mathrm{~g}$ / pot) with an increase in doses of N. Higher contents of $\mathrm{P}, \mathrm{S}, \mathrm{Ca}$, and $\mathrm{Mg}$ were observed in plants from treatments with liming, independently of the doses of $\mathrm{N}$ used. In the absence of liming the content of macronutrients in the dry matter was small, as a consequence of a smaller production of dry matter. An increase in dry matter and total alkaloids was observed when the dose of nitrogen was increased. The maximum production of total alkaloids was of $0.676 \mathrm{mg}$ of alkaloid per gram of stems and leaves dry matter that corresponds to the dose of $\mathrm{N}$ responsible for $90 \%$ of the maximum production dry matter.
\end{abstract}

Keywords: Phyllanthus niruri, mineral nutrition.

(Aceito para publicação em 04 de abril de 2.000)

\begin{abstract}
Phy hyllanthus niruri L., popularmente conhecida como quebra-pedra, é uma espécie de porte erva, encontrada na região amazônica e em algumas regiões do litoral brasileiro. Nesta planta com propriedades medicinais, foram identificadas lignanas (Row et al., 1970; Rao \& Bramley, 1971; Schneiders \& Stevenson, 1982; Singh et al., 1989; Huang et al., 1992), compostos fenólicos (Ishimaru et al., 1992) e os alcalóides norsecurinine e entnorsecirinine (Joshi et al., 1986). Estes
\end{abstract}

compostos conferem atividades hepatoprotetivas (Bhaumik \& Sharma, 1994), inibem a formação de vasos constritores (Hussain et al., 1995), inibem o vírus de hepatite $\mathrm{B}$ em marmota e pato (Thyagarajan et al., 1988) e a ligação entre o vírus HIV com a proteína VER (Qian-Cutrone et al., 1996).

Os princípios ativos das plantas medicinais podem ser influenciados pelas condições ambientes, como fertilidade do solo, $\mathrm{pH}$, umidade, temperatura e qualidade de luz. Em pH abaixo de 4,0, algumas espécies de Phyllanthus não sobreviveram mais que duas semanas (Unander \& Blumberg, 1990).

Trabalhando com Lathyrus tingitanus e Mimosa sp, Nowacki \& Waller (1975), citados por Nowacki et al. (1976) sugeriram que com o aumento da adubação nitrogenada houve síntese desproporcional do aminoácido lisina, o qual uma vez acumulado torna-se tóxico, por isso essas espécies ativam o metabolismo dos alcalóides como um tipo de adaptação. Ocorreu a con- 


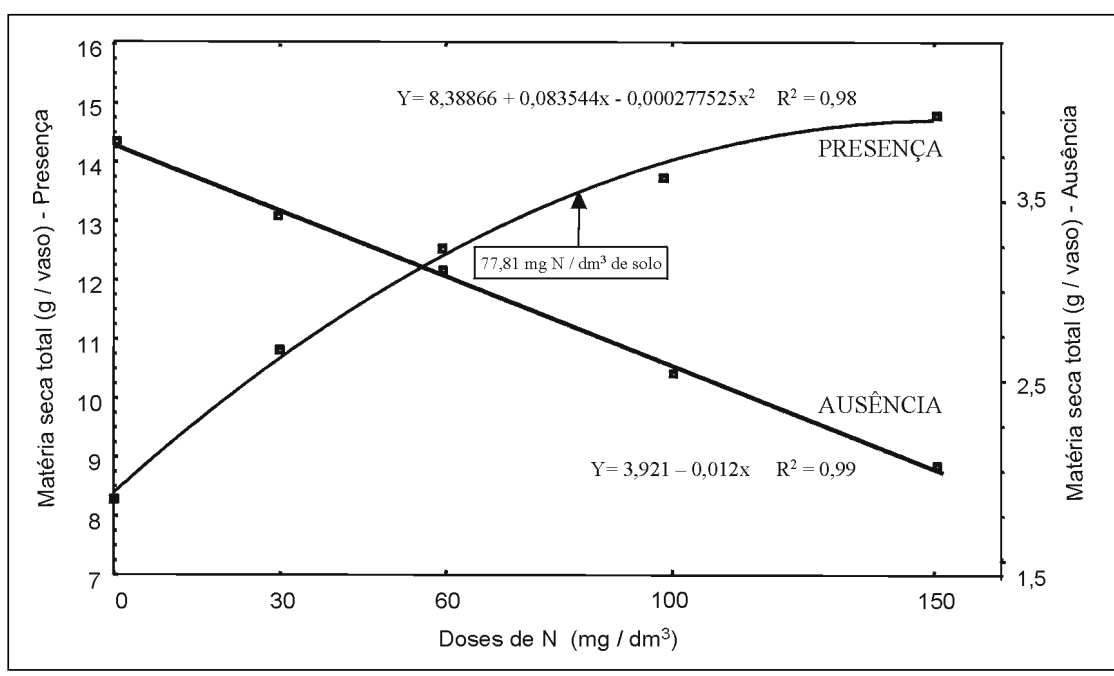

Figura 1. Matéria seca total de plantas de quebra-pedra submetidas à adubação nitrogenada, na presença e ausência de calagem. Lavras, UFLA, 1997.

versão de lisina em tingitanina e latirina em Lathyrus tingitanus e em mimosina em Mimosa sp. Por outro lado, em Atropa belladonna $\mathrm{L}$, um aumento no suprimento de N promoveu maior acúmulo de proteínas e compostos nitrogenados nos tecidos, enquanto os níveis de alcalóides encontrados foram relativamente baixos, sugerindo portanto, não haver relação direta entre alcalóides e proteínas (Schermeister et al., 1960).

O objetivo deste trabalho foi avaliar o crescimento e a produção das plantas, além do teor de alcalóides totais em quebra-pedra em resposta à calagem e adubação nitrogenada.

\section{MATERIAL E MÉTODOS}

O trabalho foi desenvolvido em casa de vegetação do Dept ${ }^{\circ}$ de Agricultura, da Universidade Federal de Lavras. As plantas foram obtidas a partir da germinação de sementes coletadas em Belém - PA, em dezembro de 1995. Foram identificadas como sendo Phyllanthus niruri L. e uma amostra foi colocada sob $n^{\circ}$ ID 84/96, no Laboratório de Botânica-Herbário na Embrapa Amazônia Oriental.

O semeio foi feito em sacos plásticos contendo solo de baixa fertilidade com areia (2:1). Aos dez dias após a emergência foram transplantadas oito mudas com aproximadamente $5,0 \mathrm{~cm}$ de altura para vasos com capacidade de 3,4 $\mathrm{dm}^{3}$ de solo, e aos sete dias após o trans- plante foi feito um desbaste, deixandose cinco plantas/vaso. O substrato utilizado foi um Cambissolo Álico, com textura média, do município de Nazareno (MG), com as seguintes características químicas: $\mathrm{pH}$ 4,7 (em água); 2,0 mg/dm 3 $\mathrm{P} ; 1,0 \mathrm{mmol} / \mathrm{dm}^{3} \mathrm{~K}^{+} ; 8,0 \mathrm{mmol} / \mathrm{dm}^{3}$ $\mathrm{Ca}^{+} ; 2,0 \mathrm{mmol}_{\mathrm{c}} / \mathrm{dm}^{3} \mathrm{Mg}^{+2}$ e 7,0 $\mathrm{mmol}_{\mathrm{c}} /$ $\mathrm{dm}^{3}$ de $\mathrm{Al}^{+3}$. Entre as características texturais encontrou-se $17 \mathrm{~g} / \mathrm{kg}$ de matéria orgânica, $660 \mathrm{~g} / \mathrm{kg}$ de areia e $170 \mathrm{~g} /$ $\mathrm{kg}$ de argila.

O experimento consistiu de cinco doses de $\mathrm{N}(0,30,60,100$ e $150 \mathrm{mg} /$ $\mathrm{dm}^{3}$ ) e o uso ou não de calcário, visando à elevação do $\mathrm{pH}$ para 6,0 , arranjados em fatorial $5 \times 2$, no delineamento experimental inteiramente casualizado, com seis repetições. Foram aplicados $\mathrm{CaCO}_{3}$ e $\mathrm{MgCO}_{3}$ na forma de sais p.a. na proporção $4: 1$ de $\mathrm{Ca}: \mathrm{Mg}$, sendo as quantidades definidas por meio de uma curva de incubação. Os vasos foram irrigados diariamente com água deionizada e tinham sua umidade reajustada a $60 \%$ do volume total de poros (VTP), que era monitorado por pesagens periódicas. $\mathrm{O}$ ciclo da cultura foi de 80 dias, sendo o final do ciclo indicado pela queda de folíolos. No momento da colheita, foram avaliados os pesos das matérias secas de partes aéreas e de raízes, além dos teores de macronutrientes e alcalóides totais da matéria seca da parte aérea das plantas.

As partes aéreas das plantas foram colhidas cortando-se rente ao solo e acondicionando-as em sacos de papel. As raízes foram cuidadosamente retiradas do solo, lavadas com água deionizada e acondicionadas em sacos de papel. Todos os materiais foram levados à estufa com aeração forçada à temperatura aproximada de $45^{\circ} \mathrm{C}$ para secagem até peso constante. A produção de matéria seca total foi obtida da soma da matéria seca das partes aéreas e de raízes.

Foram realizadas determinações de $\mathrm{N}, \mathrm{P}, \mathrm{K}, \mathrm{Ca}, \mathrm{Mg}$ e S na matéria seca da parte aérea das plantas, segundo Malavolta et al. (1997), sendo P determinado por colorimetria, $\mathrm{Ca}$ e $\mathrm{Mg}$ por espectrofotometria de absorção atômica, K por fotometria de chama e S por turbidimetria. Os teores de $\mathrm{N}$ foram determinados pelo método semi-micro Kjeldahl.

A extração dos alcalóides totais foi realizada na matéria seca da parte aérea das plantas, segundo metodologia definida por Calixto et al. (1984), nas amostras dos tratamentos onde foi realizada calagem, no laboratório do Dep. Química da UFLA. As análises de espectrofotometria de Infravermelho (IV) foram realizadas no laboratório do Departamento de Química da UFMG, em Belo Horizonte. Posteriormente, as frações obtidas das cromatografias foram analisadas em espectrofotômetro em comprimento de ondas de $270 \mathrm{~nm}$ e os alcalóides totais foram quantificados utilizando curva padrão. Os dados avaliados foram submetidos à análise por regressão polinomial.

\section{RESULTADOS E DISCUSSÃO}

A calagem e a adubação nitrogenada influenciaram no crescimento e desenvolvimento de quebra-pedra. A produção da matéria seca total (Figura 1) em solo sem calagem decresceu à medida em que se aumentaram as doses de N, demonstrando a sensibilidade dessa espécie a solos ácidos e confirmando a necessidade de calagem para o cultivo de quebra-pedra. As restrições ao crescimento do sistema radicular e da parte aérea, na ausência de calagem, provavelmente devem ter resultado do fato de terem sido afetadas a divisão e elongação celular das regiões meristemáticas (Reding \& Taylor, 
Tabela 1. Teor de macronutrientes na matéria seca da parte aérea (MSPA) de plantas de quebra-pedra submetidas a doses crescentes de N, com ou sem calagem. Lavras, UFLA, 1997.

\begin{tabular}{|c|c|c|c|c|c|c|c|}
\hline \multirow{2}{*}{$\begin{array}{c}\text { Doses de N } \\
\mathrm{mg} / \mathrm{dm}^{3}\end{array}$} & \multirow{2}{*}{ Calagem* } & $\mathbf{N}$ & $\mathbf{P}$ & $\mathrm{K}$ & $\mathrm{s}$ & $\mathrm{Ca}$ & $\mathbf{M g}$ \\
\hline & & \multicolumn{6}{|c|}{ g/kg de MSPA } \\
\hline 0 & 0 & 13,4 & 2,4 & 18,9 & 2,2 & 12,8 & 1,5 \\
\hline 30 & 0 & 23,5 & 3,4 & 25,1 & 2,7 & 13,7 & 1,9 \\
\hline 60 & 0 & 24,2 & 2,6 & 25,5 & 2,2 & 14,4 & 1,5 \\
\hline 100 & 0 & 24,8 & 2,4 & 23,7 & 1,8 & 13,8 & 1,4 \\
\hline 150 & 0 & 30,0 & 2,8 & 28,2 & 1,7 & 14,6 & 1,4 \\
\hline 0 & 1 & 13,5 & 3,7 & 14,3 & 2,3 & 15,6 & 3,5 \\
\hline 30 & 1 & 16,9 & 3,6 & 14,1 & 2,7 & 16,6 & 3,5 \\
\hline 60 & 1 & 21,9 & 3,8 & 16,2 & 2,8 & 18,3 & 3,9 \\
\hline 100 & 1 & 23,9 & 4,2 & 15,6 & 2,7 & 18,0 & 3,8 \\
\hline 150 & 1 & 25,2 & 4,0 & 16,0 & 2,7 & 17,8 & 3,5 \\
\hline \multicolumn{2}{|c|}{ DMS (1\%) - Cal $\times$ N } & 0,23 & 0,05 & 0,16 & 0,03 & 0,10 & 0,03 \\
\hline
\end{tabular}

* 0 e 1 indicam ausência e presença de calagem, respectivamente.

Tabela 2. Conteúdo de macronutrientes na matéria seca da parte aérea (MSPA) de plantas de quebra-pedra submetidas a doses crescentes de N com ou sem calagem. Lavras, UFLA, 1997.

\begin{tabular}{|c|c|c|c|c|c|c|c|}
\hline \multirow{2}{*}{$\begin{array}{c}\text { Doses de N } \\
\mathrm{mg} / \mathrm{dm}^{3}\end{array}$} & \multirow{2}{*}{ Calagem* } & $\mathbf{N}$ & $\mathbf{P}$ & $\mathbf{K}$ & $\mathbf{S}$ & $\mathrm{Ca}$ & $\mathbf{M g}$ \\
\hline & & \multicolumn{6}{|c|}{$\mathrm{mg} / \mathrm{vaso}$} \\
\hline 0 & 0 & 42,8 & 7,5 & 60,3 & 7,2 & 40,8 & 4,9 \\
\hline 30 & 0 & 73,0 & 10,7 & 78,3 & 8,4 & 42,4 & 5,8 \\
\hline 60 & 0 & 31,2 & 3,5 & 33,1 & 2,8 & 18,6 & 1,9 \\
\hline 100 & 0 & 54,8 & 5,4 & 52,5 & 4,0 & 30,6 & 3,1 \\
\hline 150 & 0 & 52,0 & 4,8 & 49,1 & 2,9 & 25,4 & 2,3 \\
\hline 0 & 1 & 99,8 & 27,6 & 105,6 & 17,1 & 115,2 & 25,7 \\
\hline 30 & 1 & 165,1 & 34,5 & 136,8 & 26,3 & 160,9 & 33,8 \\
\hline 60 & 1 & 250,2 & 43,7 & 185,5 & 32,2 & 209,5 & 45,0 \\
\hline 100 & 1 & 301,2 & 53,4 & 196,8 & 33,8 & 229,2 & 47,9 \\
\hline 150 & 1 & 343,8 & 54,4 & 217,7 & 37,1 & 243,6 & 47,8 \\
\hline \multicolumn{2}{|c|}{ DMS (1\%) - Cal x N } & 10,47 & 2,91 & 7,52 & 1,55 & 7,93 & 2,29 \\
\hline
\end{tabular}

* 0 e 1 indicam ausência e presença de calagem respectivamente.

1989). Estudando os efeitos ambientais no crescimento e produção de princípio ativo de algumas espécies de Phyllanthus, Unander \& Blumberg (1990) relataram que as plantas não sobreviveram mais que duas semanas a $\mathrm{pH}$ abaixo de 4,0 .

Com calagem (Figura 1), a dose de $\mathrm{N}$ equivalente a $90 \%$ da produção máxima média (12,25 g/vaso) de matéria seca total foi de $77,81 \mathrm{mg} / \mathrm{dm}^{3}$ de solo. Trabalhando com Phyllanthus stipulatus, em experimento de campo, Silva Filho et al. (1996) encontraram o máximo de matéria seca de $546,0 \mathrm{~g} / \mathrm{m}^{2}$ de solo, adubando com $10 \mathrm{~kg} / \mathrm{m}^{2}$ de composto orgânico. Em estudos semelhantes com Mentha arvensis, Kothari et al. (1987) encontraram $159,9 \mathrm{~kg} / \mathrm{ha}$ de $\mathrm{N}$ como a dose de máxima eficiência econômica de nitrogênio para o genótipo MA2.

As plantas tiveram maior teor dos macronutrientes $\mathrm{P}, \mathrm{S}, \mathrm{Ca}$ e $\mathrm{Mg}$ nos tratamentos onde realizou-se a calagem (Tabela 1). O mesmo não foi observado para o K, cuja maior absorção ocorreu nos tratamentos sem calagem. O nitrogênio foi absorvido em quantidades crescentes na ausência e presença de calagem. Em trabalho semelhante com mudas de jaborandi (Pilocarpus microphyllus, Starf.) Brasil (1996) en- controu a máxima produção de matéria seca com adubação nas doses $180 \mathrm{mg} /$ $\mathrm{kg}$ de $\mathrm{N}$ e $120 \mathrm{mg} / \mathrm{kg}$ de P. Estudando Artemisia annua L., Figueira et al. (1996) encontraram a máxima produção de matéria seca, de artemisinina e ácido artemisinínico, com a dose de $280 \mathrm{mg}$ / L de $\mathrm{N}$ em cultivo hidropônico.

Nos tratamentos sem calagem, o conteúdo dos macronutrientes foi menor (Tabela 2), em conseqüência da diminuição na produção de matéria seca total (Figura 1) e da crescente diminuição da absorção dos nutrientes pelas plantas, exceto de K, que apresentou absorção crescente com o aumento das doses de N. 


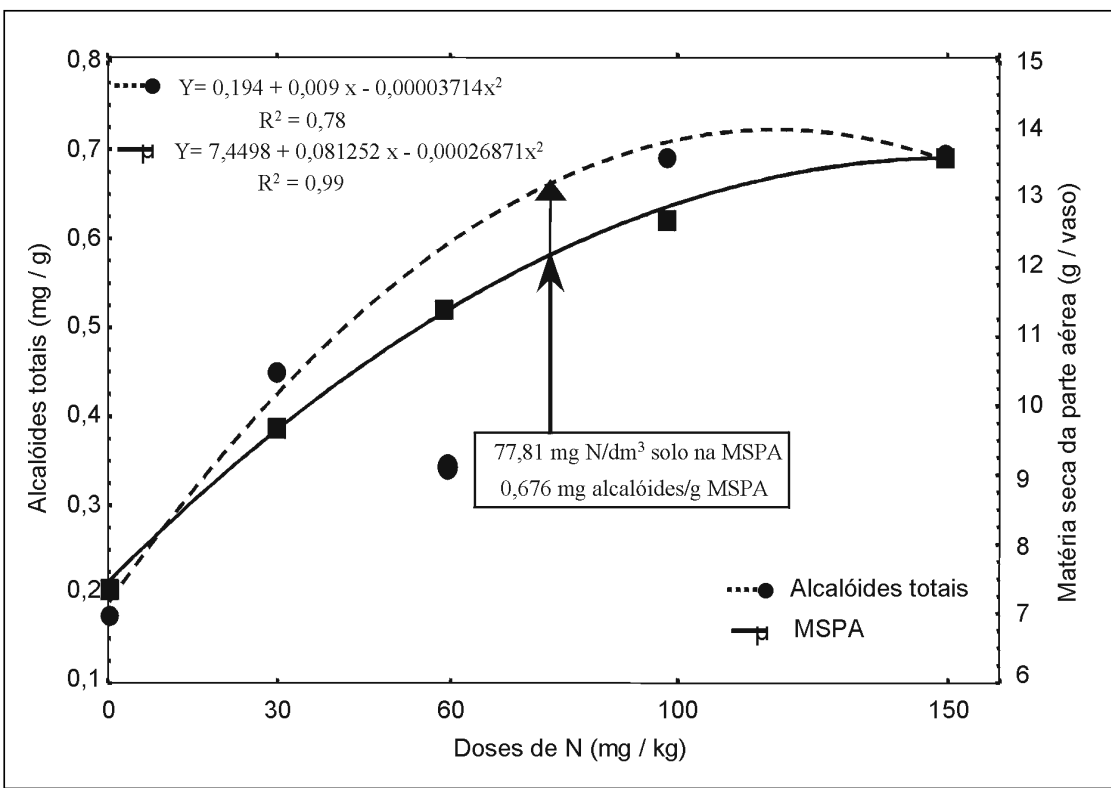

Figura 2. Alcalóides totais na matéria seca da parte aérea $(\cdot)$, matéria seca da parte aérea $(\mu)$ e produção máxima de alcalóides totais na dose de nitrogênio equivalente a $90 \%$ da produção máxima de matéria seca total das plantas de quebra-pedra submetidas à calagem e adubação nitrogenada. Lavras, UFLA, 1997.

O efeito positivo do conteúdo de $\mathrm{N}$, $\mathrm{P}, \mathrm{K}, \mathrm{S}, \mathrm{Ca}$ e Mg na matéria seca da parte aérea das plantas de quebra-pedra ocorreu principalmente nos tratamentos em que foi realizada calagem e à medida em que aumentaram-se as doses de N (Tabela 2). Este aumento no conteúdo de nutrientes foi ocasionado tanto pelo aumento nos teores (Tabela 1), como pela maior produção de biomassa pelas plantas de quebra-pedra nesta condição (Figura 1).

Observou-se aumento gradual na concentração de alcalóides na matéria seca da parte aérea à medida que aumentaram as doses de nitrogênio aplicadas (Figura 2), na presença de calagem. A produção máxima de alcalóides totais na dose de N equivalente a $90 \%$ da produção máxima de matéria seca da parte aérea $\left(77,81 \mathrm{mg} \mathrm{N} / \mathrm{dm}^{3}\right.$ solo) foi de $0,676 \mathrm{mg}$, justificando, portanto, adubação nitrogenada até certo nível, visando maior produção de matéria seca, juntamente com maior produção de alcalóides totais. Esses resultados corroboram aqueles obtidos por Nowacki et al. (1975) que investigando o efeito da disponibilidade de $\mathrm{N}$ para síntese de alcalóides em Nicotiana sp e Datura sp, encontraram acréscimo no conteúdo de $\mathrm{N}$ na matéria seca e aumento nos teores de alcalóides das plantas com o aumen- to da adubação nitrogenada. Estudando o conteúdo de alcalóides nas folhas de Tabernaemontana pachysiphon Stapf sob três concentrações de um fertilizante de liberação lenta no solo ( $\mathrm{T}_{0}=$ controle; $\mathrm{T}_{1}=3 \mathrm{~kg} / \mathrm{m}^{3}$ de Osmocote e $\mathrm{T}_{2}=6$ $\mathrm{kg} / \mathrm{m}^{3}$ de Osmocote), em casa de vegetação, Höft et al. (1996) também encontraram maior acúmulo de alcalóides indólicos sob moderada fertilização nitrogenada.

Apesar do pouco conhecimento das exigências e requerimentos nutricionais, a correção do solo e a fertilização nitrogenada mostraram-se importantes na produção de biomassa e no aumento do teor de alcalóides de quebra-pedra.

\section{AGRADECIMENTOS}

Os autores agradecem à FAPEMIG balho. A Maria de Fátima ArrigoniBlank, Ester Solange Cerqueira e Flávia Dionísio Pereira pela ajuda na instalação e condução do experimento em casa de vegetação. Ao Eng. ${ }^{\circ}$ Agrônomo Joaquim Avanir Gomes pela ajuda na identificação da espécie. Aos colegas do Laboratório de Química Orgânica e aos laboratoristas do Laboratório de Análise Foliar e do Solo da UFLA pela ajuda nas análises fitoquímicas e químicas. e FINEP pelo financiamento deste tra-

\section{LITERATURA CITADA}

BHAUMIK, A.; SHARMA, M.C. Comparative efficacy of two herbal formulations in induced hepatopathy in rabbits. Indian Journal of Animal Sciences, v. 64, n. 5, p. 465-67, 1994.

BRASIL, E.C. Níveis de nitrogênio, fósforo e potássio na produção de mudas de jaborandi. In: REUNIÃO BRASILEIRA DE FERTILIDADE DO SOLO E NUTRIÇÃO DE PLANTAS, XXII. Manaus, 1996. Anais... Manaus: SBCS, p. 666-667, 1996.

CALIXTO, J.B.; YUNES, R.A.; NETO, A.S.O.; VALLE, R.M.R.; RAE, G.A .Antispasmodic effects of an alkaloid extracted from Phyllanthus sellowianus: a comparative study with papaverine. Brazilian Journal of Medicinal Biological Research, Ribeirão Preto, v. 17, p. $313-321,1984$.

CAMARGO, O.A.; MONIZ, A.C.; JORGE, J.A.; VALADARES, J.M.A.S. Métodos de análise química, mineralógica e fisica de solos do Instituto Agronômico de Campinas. Campinas: IAC, 1986. $94 \mathrm{p}$

FIGUEIRA, G.M.; DECHEN, A.R.; CARMELLO, Q.A.C.; REHDER, V.L.G. Nutrição mineral, produção e concentração de artemisinina em Artemisia annua L. In: REUNIÃO BRASILEIRA DE FERTILIDADE DO SOLO E NUTRICÃO DE PLANTAS, XXII, 1996. Anais... Manaus: SBCS, p. 592$593,1996$.

FLÜCK, H. The influence of the soil on the content of active principles in medicinal plants. The Journal of Pharmacy and Pharmacology, v. 13, n. 7, p. 153-163, 1955.

HÖFT, M.; VERPOORTE, R.; BECK, E. Growth and alkaloid contents in leaves of Tabernaemontana pachysiphon Stapf (Apocynaceae) as influenced by light intensity, water and nutrient supply. Oecologia, n. 107, p. 160-169, 1996.

HUANG, Y.L.; CHEN, C.C.; OU, J.C Isolintetralin: a new lignan from Phyllanthus niruri. PlantaMedica, v. 58, p. 473-474, 1992.

HUSSAIN, R.A.; DICKEY, J.K.; ROSSER, M.P.; MATSON, J.A.; KOZLOWSKI, M.R. Anovel class of non-peptidic endothelin antagonists isolated from the medicinal herb Phyllanthus niruri. Journal of Natural Products, v. 58, n. 10, p. 1515-1520, 1995.

ISHIMARU, K.; YOSHIMATSU, K.; YAMAKAWA, T.; KAMADA, H.; SHIMOMURA, K. Phenolic constituents in tissue cultures of Phyllanthus niruri. Phytochemistry, v. 31, n. 6, p. 2015-2018, 1992.

JOSHI, B.S.; GAWAD, D.H.; PELLETIER, S.W.; KARTHA, G; BHANDARY, K. Isolation and structure (X-ray analysis) of ent-norsecurinine, an alkaloid from Phyllanthus niruri. Journal of Natural Products. v. 49, n. 4, p. 614-620, 1986.

KOTHARI, S.K.; SINGH, V.; SINGH, K. Response of japanese mint (Mentha arvensis) to varying levels of nitrogen application in Uttar Pradesh foot-hills. Indian Journal of Agricultural Sciences, v. 57, n. 11, p. 795-800, 1987. 
MALAVOLTA, E.; VITTI, G.C.; OLIVEIRA, S.A. Avaliação do estado nutricional das plantas: princípios e aplicações. Piracicaba: POTAFÓS, 1997. $319 \mathrm{p}$.

NOWACKI, E.; JURZYSTA, M.; GORSKI, P. Effect of availability of nitrogen on alkaloid synthesis in Solanaceae. Bulletin de LфłAcadémie Polonaise des Sciences, v. 23, n. 3, p. 219-225, 1975.

NOWACKI, E.; JURZYSTA, M.; GORSKI, P.; NOWACKA, D.; WALLER, G.R. Effect of nitrogen nutrition on alkaloid metabolism in plants. Biochemical Physiology, v. 169, p. 231240, 1976.

QIAN-CUTRONE, J.; HUANG, S.; TRIMBLE, J.; LI, H.; LIN, P-F.; ALAM, M.; KLOHR, S.E.; KADOW, K.F. Niruriside, a new HIV VER/RRE binding inhibitor from Phyllanthus niruri. Journal of Natural Products, v. 59, n. 2, p. 196-199, 1996.
RAO, G.S.; BRAMLEY, R. Hypophyllanthin. Tetrahedron Letters, n. 34, p. 3175-3178, 1971. ROW, L.R.; SATYANARAYANA, P.; SRINIVASULU, C. Crystalline constituents of Euphorbiaceae-XI. Revised structure of hypophyllanthin from Phyllanthus niruri Linn. Tetrahedron Letters, v. 26, p. 3051-3057, 1970.

REDING, V.V.; TAYLOR, H.M. Principles of soilplant interrelationships. New York: McGrawHill Publishing Company, 1989. 275 p.

SCHERMEISTER, L.J.; CRANE, F.A.; VOIGT, R.F. Nitrogenous constituents of Atropa belladonna L. grown sources of externally supplied nitrogen. Journal of the American Pharmaceutical Association, v. 49, n. 11, p. 698-705, 1960.

SCHNEIDERS, G.E.; STEVENSON, R. Structure and synthesis of the aryltetralin lignans hypophyllanthin and nirtetralin. Journal of the Chemical Society, n. 46, p. 999-1003, 1982.
SILVA FILHO, D.F.; NODA, H.; CLEMENT, C.R. ; MACHADO, F.M. O efeito da adubação orgânica na produção de biomassa em quebra-pedra em Manaus, Amazonas. Horticultura Brasileira, Brasília, v. 14, n. 1, p. 120, 1996.

SINGH, B; AGRAWAL, P.K.; THAKUR, R. A new lignan and a new neolignan from Phyllanthus niruri. Journal of Natural Products, v. 52, n. 1, p. 48-51, 1989.

THYAGARAJAN, S.P.; SUBRAMANIAN, S.; THIRUNALASUNDARI,

VENKATESWARAN, P.S. BLUMBERG, B.S. Effect of Phyllanthus amarus on chronic carriers of hepatitis B virus. The Lancet, n. 1, p. 764-766, 1988

UNANDER, D.W.; BLUMBERG, B.S. In vitro activity of Phyllanthus (Euphorbiaceae) species against the DNA polymerase of hepatitis virus: effects of growing environment and inter- and intra-specific differences. Economic Botany, v. 45, n. 2, p. 225-242, 1990. 